\title{
RANKIN'S METHOD AND JACOBI FORMS OF SEVERAL VARIABLES
}

\author{
B. RAMAKRISHNAN and BRUNDABAN SAHU ${ }^{凶}$
}

(Received 20 August 2008; accepted 5 May 2009)

Communicated by F. Calegari

\begin{abstract}
Following R. A. Rankin's method, D. Zagier computed the $n$th Rankin-Cohen bracket of a modular form $g$ of weight $k_{1}$ with the Eisenstein series of weight $k_{2}$, computed the inner product of this RankinCohen bracket with a cusp form $f$ of weight $k=k_{1}+k_{2}+2 n$ and showed that this inner product gives, up to a constant, the special value of the Rankin-Selberg convolution of $f$ and $g$. This result was generalized to Jacobi forms of degree 1 by Y. Choie and W. Kohnen. In this paper, we generalize this result to Jacobi forms defined over $\mathcal{H} \times \mathbb{C}^{(g, 1)}$.
\end{abstract}

2000 Mathematics subject classification: primary 11F50; secondary 11F60.

Keywords and phrases: Rankin's method, Jacobi forms of higher degree, Rankin-Cohen differential operators.

\section{Introduction}

There are many interesting connections between differential operators and modular forms and many interesting results have been found. In [10, 11], Rankin gave a general description of the differential operators which send modular forms to modular forms. In [6], Cohen constructed bilinear operators and obtained elliptic modular forms with interesting Fourier coefficients. In [14], Zagier studied the algebraic properties of these bilinear operators and called them Rankin-Cohen brackets. In [13], following Rankin's method, Zagier computed the $n$th Rankin-Cohen bracket of a modular form $g$ of weight $k_{1}$ with the Eisenstein series of weight $k_{2}$ and then computed the inner product of this Rankin-Cohen bracket with a cusp form $f$ of weight $k=k_{1}+k_{2}+2 n$ and showed that this inner product gives, up to a constant, the special value of the Rankin-Selberg convolution of $f$ and $g$.

Rankin-Cohen brackets for Jacobi forms were studied by Choie [2, 3] by using the heat operator. Following the aforementioned work of Zagier, Choie and Kohnen [5]

(C) 2010 Australian Mathematical Publishing Association Inc. 1446-7887/2010 \$16.00 
generalized the result of Zagier to Jacobi forms. They computed the Petersson scalar product $\left\langle f,\left[g, E_{k_{2}, m_{2}}\right]_{v}\right\rangle$ of a Jacobi cusp form $f$ of weight $k$ and index $m$ against the Rankin-Cohen bracket $\left[g, E_{k_{2}, m_{2}}\right]_{v}$ of a Jacobi form $g$ of weight $k_{1}$ and index $m_{1}$ and the Jacobi-Eisenstein series $E_{k_{2}, m_{2}}$ of weight $k_{2}$ and index $m_{2}$, where $k=$ $k_{1}+k_{2}+2 v$ and $m=m_{1}+m_{2}$. As in the case of modular forms, they expressed the inner product in terms of special values of a kind of Rankin-Selberg type convolution of the Jacobi forms $f$ and $g$, though such a convolution has not yet been studied in the case of Jacobi forms.

In this paper, we generalize the work of Choie and Kohnen to Jacobi forms defined over $\mathcal{H} \times \mathbb{C}^{(g, 1)}$. Since the method is similar, we shall give only a brief sketch of the proof with the corresponding steps.

\section{Preliminaries on Jacobi forms over $\mathcal{H} \times \mathbb{C}^{(g, 1)}$}

Fix a positive integer $g$. The Jacobi group $\Gamma_{1, g}^{J}=\Gamma_{1} \ltimes\left(\mathbb{Z}^{(g, 1)} \times \mathbb{Z}^{(g, 1)}\right)$ acts on $\mathcal{H} \times \mathbb{C}^{(g, 1)}$ in the usual way by

$$
\left(\left(\begin{array}{ll}
a & b \\
c & d
\end{array}\right),(\lambda, \mu)\right) \circ(\tau, z)=\left(\frac{a \tau+b}{c \tau+d}, \frac{z+\lambda \tau+\mu}{c \tau+d}\right),
$$

where $\Gamma_{1}=\mathrm{SL}_{2}(\mathbb{Z})$ is the full modular group. Let $k \in \mathbb{Z}$ and $M$ be a positive definite, symmetric, half-integer $g \times g$ matrix. When

$$
\gamma=\left(\left(\begin{array}{ll}
a & b \\
c & d
\end{array}\right),(\lambda, \mu)\right) \in \Gamma_{1, g}^{J}
$$

and $\phi$ is a complex-valued function on $\mathcal{H} \times \mathbb{C}^{(g, 1)}$, we define $\left.\phi\right|_{k, M} \gamma$ to be

$$
(c \tau+d)^{-k} e\left(\frac{-c}{c \tau+d} M[z+\lambda \tau+\mu]+M[\lambda] \tau+2 \lambda^{t} M z\right) \phi(\gamma \circ(\tau, z)),
$$

where for matrices $A$ and $B$ of appropriate size, $B^{t}$ denotes the transpose of $B$ and $A[B]=B^{t} A B$ and $e(a)=\exp (2 \pi i a)$.

Let $J_{k, M}$ be the space of Jacobi forms of weight $k$ and index $M$ on $\Gamma_{1, g}^{J}$, that is, the space of holomorphic functions $\phi: \mathcal{H} \times \mathbb{C}^{(g, 1)} \rightarrow \mathbb{C}$ such that $\left.\phi\right|_{k, M} \gamma=\phi$ for all $\gamma \in \Gamma_{1, g}^{J}$, which have a Fourier expansion of the form

$$
\phi(\tau, z)=\sum_{\substack{n \in \mathbb{Z}, r \in \mathbb{Z}^{g} \\ 4 n \geq M^{-1}\left[r^{t}\right]}} c(n, r) e(n \tau+r z)
$$

Further, $F$ is called a cusp form if and only if $c(n, r)=0$ when $4 n=M^{-1}\left[r^{t}\right]$. We denote the space of all Jacobi cusp forms by $J_{k, M}^{\text {cusp }}$. When $F, G \in J_{k, M}$ and one of them is a cusp form, the Petersson inner product is defined by

$$
\langle F, G\rangle=\int_{\Gamma_{1, g}^{J} \backslash \mathcal{H} \times \mathbb{C}^{(g, 1)}} F(\tau, z) \overline{G(\tau, z)} v^{k} e\left(-4 \pi M[y] \cdot v^{-1}\right) d V_{g}^{J},
$$


where $\tau=u+i v, z=x+i y$, and $d V_{g}^{J}=v^{-g-2} d u d v d x d y$ is the element of invariant measure. The space $J_{k, M}^{\text {cusp }}$ with the Petersson inner product is a finite-dimensional Hilbert space. For more details on Jacobi forms on $\mathcal{H} \times \mathbb{C}^{(g, 1)}$ we refer to [1, 15].

2.1. Poincaré series. Suppose that $n \in \mathbb{Z}, r \in \mathbb{Z}^{g}$, and $4 n>M^{-1}\left[r^{t}\right]$. When $k>$ $g+2$, let $P_{k, M ;(n, r)}$ be the $(n, r)$ th Poincaré series in $J_{k, M}^{\text {cusp }}$, characterized by

$$
\left\langle\phi, P_{k, M ;(n, r)}\right\rangle=\lambda_{k, M, D} c_{\phi}(n, r) \quad \forall \phi \in J_{k, M}^{\text {cusp }},
$$

where $c_{\phi}(n, r)$ denotes the $(n, r)$ th Fourier coefficient of $\phi$ and

$$
\lambda_{k, M, D}:=2^{\kappa(g-1)-g} \Gamma(\kappa) \pi^{-\kappa}(\operatorname{det} M)^{\kappa-1 / 2} D^{-\kappa},
$$

where

$$
\kappa=k-\frac{g}{2}-1, \quad D=\operatorname{det}(2 T), \quad T=\left(\begin{array}{cc}
n & r / 2 \\
r^{t} / 2 & M
\end{array}\right) .
$$

The Poincaré series $P_{k, M ;(n, r)}$ has the following Fourier expansion:

$$
P_{k, M ;(n, r)}(\tau, z)=\sum_{\substack{n^{\prime} \in \mathbb{Z}, r^{\prime} \in \mathbb{Z}^{g} \\ 4 n^{\prime}>M^{-1}\left[r^{\prime t}\right]}} c\left(n^{\prime}, r^{\prime}\right) e\left(n^{\prime} \tau+r^{\prime} z\right),
$$

where

$$
\begin{gathered}
c\left(n^{\prime}, r^{\prime}\right)=g_{k, M ;(n, r)}\left(n^{\prime}, r^{\prime}\right)+(-1)^{k} g_{k, M ;(n, r)}\left(n^{\prime},-r^{\prime}\right), \\
g_{k, M ;(n, r)}\left(n^{\prime}, r^{\prime}\right)=\delta_{M}\left(n, r, n^{\prime}, r^{\prime}\right)+i^{-k} \frac{2 \pi}{2^{g / 2}(\operatorname{det} M)^{1 / 2}}\left(\frac{D^{\prime}}{D}\right)^{\kappa / 2} S, \\
\delta_{M}\left(n, r, n^{\prime}, r^{\prime}\right)= \begin{cases}1 & \text { if } D=D^{\prime} \text { and } r^{\prime} \equiv r \bmod \mathbb{Z}^{g} \cdot 2 M \\
0 & \text { otherwise, }\end{cases} \\
D^{\prime}=\operatorname{det}\left(2 T^{\prime}\right), \quad T^{\prime}=\left(\begin{array}{cc}
n^{\prime} & r^{\prime} / 2 \\
r^{\prime t} / 2 & M
\end{array}\right), \\
S=\frac{1}{c^{g / 2+1}} \sum_{c \geq 1} H_{M, c}\left(n, r, n^{\prime}, r^{\prime}\right) J_{\kappa}\left(\frac{\pi \sqrt{D^{\prime} D}}{2^{g-1} \operatorname{det} M \cdot c}\right),
\end{gathered}
$$

and $H_{M, c}\left(n, r, n^{\prime}, r^{\prime}\right)$ is equal to

$$
\sum_{x, y} e_{c}\left((M[x]+r x+n) y^{-1}+n^{\prime} y+r^{\prime} x\right) e_{2 c}\left(r^{\prime} M^{-1} r^{t}\right) .
$$

In the last sum, $x$ runs over a complete set of representatives of $\mathbb{Z}^{(g, 1)}$ modulo $c \mathbb{Z}^{(g, 1)}$ while $y$ runs over a relatively prime set of representatives of $\mathbb{Z}$ modulo $c \mathbb{Z}$, and $y^{-1}$ denotes an inverse of $y$ modulo $c$. Further, $e_{c}(a):=\exp (2 \pi i a / c), a \in \mathbb{Z}$, and $J_{\kappa}$ denotes the Bessel function of order $\kappa$. For details we refer to [1, Lemma 1]. 


\section{The generalized heat operator}

For a positive definite, symmetric, half-integer $g \times g$ matrix $M=\left(m_{i j}\right)$, we define the heat operator by

$$
L_{M}:=8 \pi i|M| \frac{\partial}{\partial \tau}-\sum_{1 \leq i, j \leq g} M_{i j} \frac{\partial}{\partial z_{i}} \frac{\partial}{\partial z_{j}},
$$

where $\tau \in \mathcal{H}$ and $z^{t}=\left(z_{1}, z_{2}, \ldots, z_{g}\right) \in \mathbb{C}^{g}$, while $|M|=\operatorname{det} M$ and $M_{i j}$ is the cofactor of $m_{i j}$. Note that when $g=1$ the heat operator above reduces to the classical heat operator, namely, $8 \pi i m \partial / \partial \tau-\partial^{2} / \partial z^{2}$. Let $r^{t}=\left(r_{1}, r_{2}, \ldots, r_{g}\right)$. Then using the fact that

$$
\begin{aligned}
\frac{\partial}{\partial \tau}(e(n \tau+r z)) & =2 \pi i n e(n \tau+r z), \\
\frac{\partial}{\partial z_{\alpha}}(e(n \tau+r z)) & =2 \pi i r_{\alpha} e(n \tau+r z), \\
\frac{\partial}{\partial z_{\alpha}} \frac{\partial}{\partial z_{\beta}}(e(n \tau+r z)) & =(2 \pi i)^{2} r_{\alpha} r_{\beta} e(n \tau+r z),
\end{aligned}
$$

where $1 \leq \alpha, \beta \leq g$, we find that $L_{M}(e(n \tau+r z))$ is equal to

$$
\begin{gathered}
8 \pi i|M| \cdot 2 \pi i n \cdot e(n \tau+r z)-\sum_{1 \leq \alpha, \beta \leq g} M_{\alpha \beta}(2 \pi i)^{2} r_{\alpha} r_{\beta} e(n \tau+r z) \\
=(2 \pi i)^{2}\left(4 n|M|-\tilde{M}\left[r^{t}\right]\right) e(n \tau+r z),
\end{gathered}
$$

where $\tilde{M}$ denotes the matrix of cofactors $M_{i j}$ of the symmetric matrix $M$. The next lemma describes the action of the heat operator on Jacobi forms.

LeMmA 3.1. Let $F \in J_{k, M}$. Then

$$
\left.\left(L_{M} F\right)\right|_{k+2, M} A=L_{M}\left(\left.F\right|_{k, M} A\right)+8 \pi i|M|(\kappa+1)\left(\frac{\gamma}{\gamma \tau+\delta}\right)\left(\left.F\right|_{k, M} A\right),
$$

for all $A \in \Gamma_{1}$ of the form $\left(\begin{array}{ll}* & * \\ \gamma & \delta\end{array}\right)$. In general, for any positive integer $v$,

$$
\begin{aligned}
& \left.\left(L_{M}^{v} F\right)\right|_{k+2 v, M} A \\
& \quad=\sum_{l=0}^{v}\left(\begin{array}{l}
v \\
l
\end{array}\right)(8 \pi i|M|)^{v-l} \frac{(\kappa+v) !}{(\kappa+l) !}\left(\frac{\gamma}{\gamma \tau+\delta}\right)^{v-l} L_{M}^{l}\left(\left.F\right|_{k, M} A\right) .
\end{aligned}
$$

Moreover, for all $\lambda, \lambda^{\prime} \in \mathbb{Z}^{g}$,

$$
L_{M}\left(\left.F\right|_{M}\left[\lambda, \lambda^{\prime}\right]\right)=\left.\left(L_{M} F\right)\right|_{M}\left[\lambda, \lambda^{\prime}\right] .
$$

PROOF. Though our $L_{M}$ operator differs slightly from the operator defined in [4], the proof goes along the same lines as [4, proof of Lemma 3.3]. 
We now define the Rankin-Cohen bracket for Jacobi forms on $\mathcal{H} \times \mathbb{C}^{(g, 1)}$.

Definition 3.2. Suppose that $F \in J_{k_{1}, M_{1}}$ and $G \in J_{k_{2}, M_{2}}$, where $k_{1}$ and $k_{2}$ are positive integers and $M_{1}$ and $M_{2}$ are positive definite, symmetric, half-integer $g \times g$ matrices, and that $v$ is a nonnegative integer. Define the $v$ th Rankin-Cohen bracket of $F$ and $G$ by

$$
[F, G]_{v}=\sum_{l=0}^{v}(-1)^{l}\left(\begin{array}{c}
\kappa_{1}+v \\
v-l
\end{array}\right)\left(\begin{array}{c}
\kappa_{2}+v \\
l
\end{array}\right)\left|M_{1}\right|^{v-l}\left|M_{2}\right|^{l} L_{M_{1}}^{l}(F) L_{M_{2}}^{v-l}(G),
$$

where $\kappa_{j}=k_{j}-g / 2-1$ when $j=1,2$.

Using Lemma 3.1, we show that the Rankin-Cohen bracket $[,]_{\nu}$ gives a bilinear map from $J_{k_{1}, M_{1}} \times J_{k_{2}, M_{2}}$ to $J_{k_{1}+k_{2}+2 v, M_{1}+M_{2}}$ (in fact, into the space of cusp forms if $v>0)$.

Proposition 3.3. Suppose that $F \in J_{k_{1}, M_{1}}, G \in J_{k_{2}, M_{2}}$, and that $v$ is a nonnegative integer. Then $[F, G]_{v} \in J_{k_{1}+k_{2}+2 v, M_{1}+M_{2}}$. Furthermore, if $v$ is positive, then $[F, G]_{v} \in J_{k_{1}+k_{2}+2 v, M_{1}+M_{2}}^{\text {cusp }}$.

PROOF. By (3.2), we see that the action of the heat operator on Jacobi forms is invariant under the lattice action and so the invariance of the Rankin-Cohen bracket with respect to the lattice action follows from the definition. It remains to show that the Rankin-Cohen bracket is invariant under the stroke operation, with respect to the group action. Making use of (3.1), we see that if $A=\left(\begin{array}{ll}* & * \\ c & d\end{array}\right) \in \Gamma_{1}$,

$$
\begin{aligned}
{\left.[F, G]_{v}\right|_{k_{1}+k_{2}+2 v, M_{1}+M_{2}} A } \\
\quad=\left.\left.\sum_{l=0}^{v} d\left(\kappa_{1}, \kappa_{2}, l, v\right) L_{M_{1}}^{l}(F)\right|_{k_{1}+2 l, M_{1}} A L_{M_{2}}^{v-l}(G)\right|_{k_{2}+2(v-l), M_{2}} A \\
\quad=\sum_{l=0}^{v} d\left(\kappa_{1}, \kappa_{2}, l, v\right) S\left(\kappa_{1}, \kappa_{2}, l, v\right),
\end{aligned}
$$

where

$$
d\left(\kappa_{1}, \kappa_{2}, l, v\right)=(-1)^{l}\left(\begin{array}{c}
\kappa_{1}+v \\
v-l
\end{array}\right)\left(\begin{array}{c}
\kappa_{2}+v \\
l
\end{array}\right)\left|M_{1}\right|^{v-l}\left|M_{2}\right|^{l},
$$

and $S\left(\kappa_{1}, \kappa_{2}, l, v\right)$ is equal to

$$
\begin{aligned}
& \sum_{u=0}^{l} \sum_{v=0}^{v-l}\left(\begin{array}{l}
l \\
u
\end{array}\right)\left(\begin{array}{c}
v-l \\
v
\end{array}\right)(8 \pi i)^{v-u-v}\left|M_{1}\right|^{v-u}\left|M_{2}\right|^{v-v} \\
& \quad \times \frac{\left(\kappa_{1}+l\right) !}{\left(\kappa_{1}+u\right) !} \frac{\left(\kappa_{2}+v-l\right) !}{\left(\kappa_{2}+v\right) !}\left(\frac{c}{c \tau+d}\right)^{v-u-v} L_{M_{1}}^{u}(F) L_{M_{2}}^{v}(G) .
\end{aligned}
$$

The terms on the right-hand side for which $u+v=v$ give $[F, G]_{v}$, and so it remains to show that the terms vanish when $u+v<v$. When $u+v<v$ and $u \leq v$, the 
coefficient of $L_{M_{1}}^{u}(F) L_{M_{2}}^{v}(G)$ is given by

$$
\begin{aligned}
& \left(\frac{8 \pi i c}{c \tau}+d\right)^{v-u-v}\left|M_{1}\right|^{v-u}\left|M_{2}\right|^{v-v} \frac{\left(\kappa_{1}+v\right) !}{u !\left(\kappa_{1}+u\right) !} \frac{\left(\kappa_{2}+v\right) !}{v !\left(\kappa_{2}+v\right) !} \\
& \quad \times \sum_{l=u}^{v-v} \frac{(-1)^{l}}{(l-u) !(v-v-l) !}
\end{aligned}
$$

and the sum in the last expression is equal to zero. This completes the proof.

We shall now state the main theorem of this paper.

THEOREM 3.4. Suppose that $F \in J_{k, M}^{\text {cusp }}$, with Fourier coefficients $a(n, r)$, and $G \in$ $J_{k_{1}, M_{1}}$, with Fourier coefficients $b(n, r)$. Suppose that $M=M_{1}+M_{2}$ and that $k=k_{1}+k_{2}+2 v$, where $v \geq 0, k_{1}>g+2$ and $k_{2}>k_{1}+g+2$, and let $E_{k_{2}, M_{2}}$ be the Jacobi-Eisenstein series in $J_{k_{2}, M_{2}}$. Then

$$
\begin{aligned}
& \left\langle F,\left[G, E_{k_{2}, M_{2}}\right]_{\nu}\right\rangle \\
& =c_{k, k_{2}, M, M_{2}, g ; v} \sum_{\substack{n \in \mathbb{Z}, r \in \mathbb{Z}^{g} \\
4 n \geq M_{1}^{-1}\left[r^{t}\right]}} \frac{\left(4 n\left|M_{1}\right|-\tilde{M}_{1}\left[r^{t}\right]\right)^{v} a(n, r) \overline{b(n, r)}}{\left(4 n|M|-\tilde{M}\left[r^{t}\right]\right)^{\kappa}},
\end{aligned}
$$

where

$$
c_{k, k_{2}, M, M_{2}, g ; v}=2^{\kappa(g-1)-g-2 v} \pi^{-\kappa-2 v}|M|^{\kappa-1 / 2}\left|M_{2}\right|^{-v} \Gamma(\kappa) \frac{v ! \kappa_{2} !}{\left(\kappa_{2}+v\right) !},
$$

$\kappa=k-g / 2-1$ and $\kappa_{2}=k_{2}-g / 2-1$.

The rest of this section is devoted to a proof of Theorem 3.4.

3.1. The action of the heat operator on Eisenstein series. Let $E_{k, M}$ be the JacobiEisenstein series of weight $k$ and index $M$, defined by

$$
E_{k, M}=\left.\sum_{\gamma \in \Gamma_{1, g, \infty}^{J} \backslash \Gamma_{1, g}^{J}} 1\right|_{k, M} \gamma
$$

where

$$
\Gamma_{1, g, \infty}^{J}=\left\{\left(\left(\begin{array}{ll}
1 & a \\
0 & 1
\end{array}\right),(0, \mu)\right) \mid a \in \mathbb{Z}, \mu \in \mathbb{Z}^{g}\right\} .
$$

LEMMA 3.5. Suppose that $v$ is a positive integer. Then $\left(L_{M}^{v} E_{k, M}\right)(\tau, z)$ is equal to

$$
\begin{aligned}
& (-4|M|)^{\nu} \frac{\Gamma(\kappa+1+v)}{\Gamma(\kappa+1)} \\
& \times \sum_{\substack{\lambda \in \mathbb{Z}^{g} \\
\left(\begin{array}{l}
a \\
c
\end{array}\right) \in \Gamma_{\infty} \backslash \Gamma_{1}}} \frac{(2 \pi i c)^{v}}{(c \tau+d)^{k+v}} e\left(M[\lambda] \frac{a \tau+b}{c \tau+d}+\frac{2 \lambda^{t} M z}{c \tau+d}-\frac{c M[z]}{c \tau+d}\right) .
\end{aligned}
$$


PROOF. Using the definition of the Eisenstein series,

$$
L_{M}^{v} E_{k, M}=\sum_{\gamma \in \Gamma_{1, g, \infty}^{J} / \Gamma_{1, g}^{J}} L_{M}^{\nu}\left(\left.1\right|_{k, M} \gamma\right)
$$

By taking a set of coset representatives $\left(\left(\begin{array}{ll}a & b \\ c & d\end{array}\right),(a \lambda, b \lambda)\right)$ in the sum above, where $\left(\begin{array}{ll}a & b \\ c & d\end{array}\right) \in \Gamma_{\infty} \backslash \Gamma_{1}$ and $\lambda \in \mathbb{Z}^{g}$,

$$
L_{M}^{v} E_{k, M}=\left.\sum_{\substack{\lambda \in \mathbb{Z}^{g} \\
\left(\begin{array}{ll}
a & b \\
c & d
\end{array}\right) \in \Gamma_{\infty} \backslash \Gamma_{1}}} L_{M}^{v}\left(\left.1\right|_{k, M}\left(\begin{array}{ll}
a & b \\
c & d
\end{array}\right)\right)\right|_{k, M}(a \lambda, b \lambda)
$$

It is easy to see that

$$
\begin{aligned}
L_{M}\left(\left.1\right|_{k, M}\left(\begin{array}{ll}
a & b \\
c & d
\end{array}\right)\right) & =L_{M}\left((c \tau+d)^{-k} e\left(\frac{-c M[z]}{c \tau+d}\right)\right) \\
& =-8 \pi i c|M|(\kappa+1)(c \tau+d)^{-k-1} e\left(\frac{-c M[z]}{c \tau+d}\right),
\end{aligned}
$$

where we have used the fact that

$$
\sum_{1 \leq \alpha, \beta \leq g} M_{\alpha \beta}\left(\sum_{1 \leq i \leq g} m_{i \beta} z_{i}\right)\left(\sum_{1 \leq i \leq g} m_{i \alpha} z_{i}\right)=|M| \sum_{1 \leq \alpha, \beta \leq g} m_{\alpha \beta} z_{\alpha} z_{\beta},
$$

and

$$
\sum_{1 \leq \alpha, \beta \leq g} M_{\alpha \beta} m_{\alpha \beta}=g|M|
$$

Therefore

$$
\begin{aligned}
& L_{M}^{v}\left((c \tau+d)^{-k} e\left(\frac{-c M[z]}{c \tau+d}\right)\right) \\
& \quad=(-4)^{v} \frac{\Gamma(\kappa+v+1)}{\Gamma(\kappa+1)}|M|^{v}(2 \pi i c)^{v}(c \tau+d)^{-k-v} e\left(\frac{-c M[z]}{c \tau+d}\right) .
\end{aligned}
$$

Since

$$
\begin{aligned}
(c \tau & +d)\left.^{-k-v} e\left(\frac{-c M[z]}{c \tau+d}\right)\right|_{k, M}(a \lambda, b \lambda) \\
& =(c \tau+d)^{-k-v} e\left(M[\lambda] \frac{a \tau+b}{c \tau+d}+\frac{2 \lambda^{t} M z}{c \tau+d}-\frac{c M[z]}{c \tau+d}\right),
\end{aligned}
$$

the required result follows. 
3.2. A representation of $[G, E]_{v}$ in terms of the Poincaré series. We first obtain a growth estimate for the Fourier coefficients of a Jacobi form. We use Landau notation: for a positive valued function $h$, we write $f \ll h$ to mean that there exists a constant (called the 'implied constant') $C$ such that $|f| \leq C h$.

LeMma 3.6. Suppose that $k>g+2$ and that $F \in J_{k, M}$ has Fourier coefficients $c(n, r)$. Put $D_{1}=\sum_{i, j} M_{i j} r_{i} r_{j}-4 n|M|$. Then

$$
c(n, r) \ll\left|D_{1}\right|^{k-g / 2-1}=\left|D_{1}\right|^{\kappa},
$$

if $D_{1}<0$. Moreover, if $F$ is a cusp form, then

$$
c(n, r) \ll\left|D_{1}\right|^{k / 2-g / 2} .
$$

The implied constants in these inequalities depend only on $k, g$ and $|M|$.

PROOF. If $F$ is a cusp form, then the required estimate was proved by Böcherer and Kohnen [1]. If $F$ is not a cusp form, then it can be written as a linear combination of the Eisenstein series $E_{k, M}$ and a cusp form. We now show that $e_{k, M}(n, r)$, the $(n, r)$ th Fourier coefficient of $E_{k, M}$, satisfies the estimate (3.5), from which the lemma follows. Taking the same set of coset representatives as in the proof of the lemma above, we see that $E_{k, M}$ is equal to

$$
\begin{aligned}
& \left.\sum_{\gamma \in \Gamma_{1, g, \infty}^{J} \backslash \Gamma_{1, g}^{J}} 1\right|_{k, M} \gamma \\
& =\left.\frac{1}{2} \sum_{\substack{\lambda \in \mathbb{Z}^{g} \\
c, d \in \mathbb{Z} \\
(c, d)=1}} 1\right|_{k, M}\left(\left(\begin{array}{ll}
a & b \\
c & d
\end{array}\right),(a \lambda, b \lambda)\right) \\
& =\frac{1}{2} \sum_{\substack{\lambda \in \mathbb{Z}^{g} \\
c, d \in \mathbb{Z} \\
(c, d)=1}}(c \tau+d)^{-k} e\left(\frac{-c}{c \tau+d} M[z+a \lambda \tau+b \lambda]+M[a \lambda] \tau+2 a \lambda^{t} M z\right) \\
& =\frac{1}{2} \sum_{\substack{\lambda \in \mathbb{Z}^{g} \\
c, d \in \mathbb{Z} \\
(c, d)=1}}(c \tau+d)^{-k} e\left(M[\lambda] \frac{a \tau+b}{c \tau+d}+\frac{2 \lambda^{t} M z}{c \tau+d}-c \frac{M[z]}{c \tau+d}\right) .
\end{aligned}
$$

Proceeding in the usual way, splitting the sum into the part where $c=0$ and the part where $c \neq 0$, we see that $E_{k, M}(\tau, z)$ is equal to

$$
\sum_{\lambda \in \mathbb{Z}^{g}} e\left(M[\lambda] \tau+2 \lambda^{t} M z\right)+\sum_{c=1}^{\infty} c^{-k} \sum_{\substack{d \\(d, c)=1 \\ \lambda \bmod c}} e\left(\frac{a}{c} M[\lambda]\right) F_{k, M}\left(\tau+\frac{d}{c}, z-\frac{\lambda}{c}\right),
$$


where

$$
F_{k, M}(\tau, z)=\sum_{p \in \mathbb{Z}, q \in \mathbb{Z}^{g}}(\tau+p)^{-k} e(-M[z+q] /(\tau+p)) .
$$

Using the Poisson summation formula, the $(n, r)$ th Fourier coefficient of $F_{k, M}(\tau, z)$ is given by

$$
\gamma(n, r)= \begin{cases}0 & \text { if } \tilde{M}\left[r^{t}\right] \geq 4 n|M|, \\ \alpha_{k, g}|M|^{-1 / 2}\left(\frac{2 \pi i}{4|M|}\left(4 n|M|-\tilde{M}\left[r^{t}\right]\right)\right)^{\kappa} & \text { if } \tilde{M}\left[r^{t}\right]<4 n|M|,\end{cases}
$$

where

$$
\alpha_{k, g}=\left(\frac{1}{2 i}\right)^{g / 2} \frac{\pi \operatorname{cosec}(\pi(\kappa+1))}{\Gamma(\kappa+1)} .
$$

Plugging in this Fourier coefficient and estimating the Gauss sum, we get

$$
e_{k, M}(n, r) \ll\left|D_{1}\right|^{\kappa},
$$

where the implied constant depends only on $k, g$ and $|M|$.

We need the following lemma, which gives the absolute convergence of a series that is required to get an expression of the Rankin-Cohen bracket of $F$ with the Eisenstein series in terms of the Poincaré series. For given positive $\epsilon$ and $C$, define the set $V_{\epsilon, C}$ to be

$$
\left\{(\tau, z) \in \mathcal{H} \times \mathbb{C}^{g}: v \geq \epsilon,\left|y_{j} v^{-1}\right| \leq C,\left|x_{j}\right| \leq 1 / \epsilon, u \leq 1 / \epsilon, \forall j=1,2, \ldots, g\right\} .
$$

LEMMA 3.7. The series

$$
\left.v^{k} \exp (-2 \pi M[y] / v) \sum_{\substack{n \in \mathbb{Z}, r \in \mathbb{Z}^{g} \\ 4 n \geq M_{1}^{-1}\left[r^{t}\right] \\ \gamma \in \Gamma_{1, \infty}^{J} \backslash \Gamma_{1}^{J}}}\left(4 n\left|M_{1}\right|-\tilde{M}_{1}\left[r^{t}\right]\right) e(n \tau+r z)\right|_{k, M} \gamma
$$

(where $\tau=u+i v, z_{j}=x_{j}+i y_{j}$, and $\left.y=\left(y_{1}, y_{2}, \ldots, y_{g}\right)^{t}\right)$ is absolutely uniformly convergent on the subsets $V_{\epsilon, C}$.

PROOF. Using Lemma 3.6, it is sufficient to prove the uniform convergence of the series

$$
v^{k} \exp (-2 \pi M[y] / v) \sum_{\substack{n \in \mathbb{Z}, r \in \mathbb{Z}^{g} \\ 4 n \geq M_{1}^{-1}\left[r^{t}\right] \\ \gamma \in \Gamma_{1, \infty}^{J} / \Gamma_{1}^{J}}}\left(4 n\left|M_{1}\right|-\tilde{M}_{1}\left[r^{t}\right]\right)^{\nu+\kappa_{2}}|e(n \tau+r z)|_{k, M} \gamma(\tau, z) \mid
$$

in the given ranges. Take $\tau^{\prime} \in \mathcal{H}_{g}$ such that $Z=\left(\begin{array}{cc}\tau & z \\ z & \tau\end{array}\right)^{\prime} \in \mathcal{H}_{g+1}$. Also let $T=\left(\begin{array}{cc}n & r^{t} / 2 \\ r / 2 & M\end{array}\right)$. By the assumption that $4 n \geq M_{1}^{-1}\left[r^{t}\right]$, we see that $T$ is positive semidefinite. Now we 
embed $\Gamma_{1, g}^{J}=\mathrm{SL}_{2}(\mathbb{Z}) \ltimes\left(\mathbb{Z}^{g} \times \mathbb{Z}^{g}\right)$ into $\Gamma_{g+1}$ (denoting the embedding by $\gamma \mapsto \gamma^{*}$ ) defined by combining the following two embeddings:

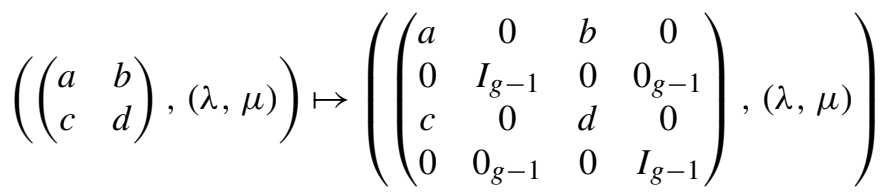

and

$$
\left(\left(\begin{array}{cc}
A & B \\
C & D
\end{array}\right),(\lambda, \mu)\right) \mapsto\left(\begin{array}{cccc}
A & 0 & B & \mu^{\prime} \\
\lambda & 1 & \mu & 0 \\
C & 0 & D & -\lambda^{\prime} \\
0 & 0 & 0 & 1
\end{array}\right)
$$

where $\left(\lambda^{\prime t}, \mu^{\prime t}\right)=(\lambda, \mu)\left(\begin{array}{ll}A & B \\ C & D\end{array}\right)^{-1}$. We have

$$
\left(\left.e(n \tau+r z)\right|_{k, M \gamma}\right)(\tau, z)=\exp \left(-2 \pi i m \tau^{\prime}\right)\left(\left.e(\operatorname{tr}(T Z))\right|_{k} \gamma^{*}\right)(Z)
$$

where $\left.\right|_{k}$ (on the right-hand side) is the usual stroke operation on functions $F$ : $\mathcal{H}_{g+1} \rightarrow \mathbb{C}$, and we can view the sum of absolute terms of the $(n, r)$ th Poincaré series as a subseries of the sum of absolute terms of the $T$ th Poincare series on $\Gamma_{g+1}$. Suppose that $(\tau, z) \in V_{\epsilon, C}$. Then by taking $\tau^{\prime}=i \min _{1 \leq j \leq g}\left\{\left(y_{j}^{2} / v+\delta\right)\right\}$ with $\delta>0$, we see that $Z=\left(\begin{array}{cc}\tau & z \\ z & \tau^{\prime}\end{array}\right) \in \mathcal{H}_{g+1}$ where $Y=\operatorname{Im} Z>\epsilon^{\prime} / 2$ for some $\epsilon^{\prime}$ depending on $\epsilon, C$ and $\delta$. Since the sum of the absolute terms of the $T$ th Poincare series on the subsets where $Y \geq \epsilon^{\prime} I_{g}$ and $\operatorname{tr}\left(X^{\prime} X\right) \leq 1 / \epsilon^{\prime}$ (up to some constants) is majorized by that sum evaluated at an arbitrary single point $Z_{0}$ (see [9]), it is sufficient to take $Z_{0}=i I_{g}$ and prove the convergence of the series above at $(\tau, z)=(i, 0, \ldots, 0)$, that is, using the the coset representation, the convergence of the series

$$
\begin{gathered}
\sum_{\substack{n \in \mathbb{Z}, r \in \mathbb{Z}^{g}, 4 n>M_{1}^{-1}\left[r^{t}\right] \\
(c, d)=1, \lambda \in \mathbb{Z}^{g}}} \frac{\left(4 n\left|M_{1}\right|-\tilde{M}_{1}\left[r^{t}\right]\right)^{v+\kappa_{2}}}{\left|(c i+d)^{k}\right|}\left|\exp \left(2 \pi i\left(n \frac{a i+b}{c i+d}+r \frac{a \lambda i+b \lambda}{c i+d}\right)\right)\right| \\
\times\left|\exp \left(2 \pi i\left(\frac{-c}{c i+d} M[a \lambda i+b \lambda]+M[a \lambda]\right)\right)\right|
\end{gathered}
$$

Now proceeding as in [5], we get the required convergence with the assumption that $k_{2}>k_{1}+g+2$.

Proposition 3.8. Let $k, k_{1}, k_{2}, M, M_{1}$, and $M_{2}$ be as in Theorem 3.4. Take $G \in J_{k_{1}, M_{1}}$ with Fourier expansion

$$
G(\tau, z)=\sum_{\substack{n \in \mathbb{Z}, r \in \mathbb{Z}^{g} \\ 4 n \geq M_{1}^{-1}\left[r^{t}\right]}} b(n, r) e(n \tau+r z) .
$$


Then

$$
\left[G, E_{k_{2}, M_{2}}\right]_{v}=c_{k_{1}, k_{2}, M_{1}, M_{2}, g ; \nu} \sum_{\substack{n \in \mathbb{Z}, r \in \mathbb{Z}^{g} \\ 4 n \geq M_{1}^{-1}\left[r^{t}\right]}}\left(4 n\left|M_{1}\right|-\tilde{M}_{1}\left[r^{t}\right]\right)^{v} b(n, r) P_{k, M ;(n, r)},
$$

where

$$
c_{k_{1}, k_{2}, M_{1}, M_{2}, g ; v}=(2 \pi)^{-2 v}\left|M_{2}\right|^{-v} \frac{v ! \kappa_{2} !}{\left(\kappa_{2}+v\right) !} .
$$

PROOF. Using the definition of the Poincare series, the action of the heat operator on Fourier coefficients, and by the absolute convergence (obtained in Lemma 3.7) we see that the series on the right-hand side of (3.6) can be written as

$$
\begin{aligned}
\sum_{\substack{\gamma \in \Gamma_{1, g, \infty}^{J} \backslash \Gamma_{1, g}^{J} \\
\times}}\left(\left.1\right|_{k, M} \gamma\right)(\tau, z) \\
\sum_{\substack{n \in \mathbb{Z}, r \in \mathbb{Z}^{g} \\
4 n \geq M_{1}^{-1}\left[r^{t}\right]}}\left(4 n\left|M_{1}\right|-\tilde{M}_{1}\left[r^{t}\right]\right)^{v} b(n, r) e(n \tau+r z)(\gamma \circ(\tau, z)) \\
=\sum_{\gamma \in \Gamma_{1, g, \infty}^{J} \backslash \Gamma_{1, g}^{J}}\left(\left.1\right|_{k, M} \gamma\right)(\tau, z) \cdot(2 \pi i)^{-2 v}\left(L_{M_{1}}^{v} G\right)(\gamma \circ(\tau, z)) \\
=\left.\frac{1}{(2 \pi i)^{2 v}} \sum_{\gamma \in \Gamma_{1, g, \infty}^{J} \backslash \Gamma_{1, g}^{J}}\left(\left.1\right|_{k_{2}, M_{2}} \gamma\right)(\tau, z)\left(L_{M_{1}}^{v} G\right)\right|_{k_{1}+2 v, M_{1}} \gamma(\tau, z) .
\end{aligned}
$$

By taking the same set of representatives for the sum over $\gamma$ as in the proof of Lemma 3.5, and using the fact that $G \in J_{k, M_{1}}$, we get

$$
\begin{gathered}
\left.\frac{1}{(2 \pi i)^{2 v}} \sum_{\gamma \in \Gamma_{1, g, \infty}^{J} \backslash \Gamma_{1, g}^{J}}\left(\left.1\right|_{k_{2}, M_{2}} \gamma\right)(\tau, z)\left(L_{M_{1}}^{v} G\right)\right|_{k_{1}+2 v, M_{1}} \gamma(\tau, z) \\
=\frac{1}{(2 \pi i)^{2 v}} \sum_{l=0}^{v} 4^{v-l} \frac{\left(\kappa_{1}+v\right) !}{\left(\kappa_{1}+l\right) !}\left(\begin{array}{l}
v \\
l
\end{array}\right)\left|M_{1}\right|^{v-l} L_{M_{1}}^{l}(G)(\tau, z) S,
\end{gathered}
$$

where $S$ is equal to

$$
\sum_{\substack{\lambda \in \mathbb{Z}^{g} \\
\left(\begin{array}{l}
a \\
c
\end{array}\right) \in \Gamma_{\infty} \backslash \Gamma_{1}}} \frac{(\pi i c)^{\nu-l}}{(c \tau+d)^{\nu-l+k_{2}}} e\left(\frac{-c M_{2}[z+a \lambda \tau+b \lambda]}{c \tau+d}+M_{2}[a \lambda]+2(a \lambda)^{t} M_{2} z\right) .
$$

Using Lemma 3.5, the inner sum $S$ in the expression above is equal to

$$
(-4)^{-(v-l)} \frac{\kappa_{2} !}{\left(\kappa_{2}+v-l\right) !}\left|M_{2}\right|^{l} L_{M_{2}}^{\nu-l} E_{k_{2}, M_{2}}(\tau, z),
$$


so we conclude that the sum on the right-hand side of (3.6) is equal to

$$
\begin{aligned}
(2 \pi)^{-2 v} & \sum_{l=0}^{v}(-1)^{l} \frac{\left(\kappa_{1}+v\right) ! \kappa_{2} !}{\left(\kappa_{1}+l\right) !\left(\kappa_{2}+v-l\right) !}\left(\begin{array}{l}
v \\
l
\end{array}\right)\left|M_{1}\right|^{v-l}\left|M_{2}\right|^{l-v} \\
& \times L_{M_{1}}^{l}(G)(\tau, z) L_{M_{2}}^{\nu-l} E_{k_{2}, M_{2}}(\tau, z) .
\end{aligned}
$$

The proof is now complete.

3.3. Proof of Theorem 3.4. We first observe that by Lemma 3.6 the series on the right-hand side of (3.3) is absolutely convergent and hence is majorized by

$$
\sum_{\substack{n \geq 1, r \in \mathbb{Z}^{g} \\ 4 n \geq M_{1}^{-1}\left[r^{t}\right]}} \frac{\left(4 n\left|M_{1}\right|-\tilde{M}_{1}\left[r^{t}\right]\right)^{\kappa_{1}+v}}{\left(4 n|M|-\tilde{M}\left[r^{t}\right]\right)^{k / 2-1}} \ll \sum_{n \geq 1} \frac{n^{g / 2} \cdot n^{\kappa_{1}+v}}{n^{k / 2-1}}=\sum_{n \geq 1} \frac{1}{n^{\left(k_{2}-k_{1}\right) / 2}}<\infty
$$

since $k=k_{1}+k_{2}+2 v$ and $k_{2}>k_{1}+g+2$ by assumption. The standard fundamental domain for the action of $\Gamma_{1, g}^{J}$ on $\mathcal{H} \times \mathbb{C}^{(g, 1)}$ is contained in one of the sets $V_{\epsilon, C}$ occurring in the statement of Lemma 3.7. Therefore, using Lemma 3.7, we deduce from Proposition 3.8 that $\left\langle F,\left[G, E_{k_{2}, M_{2}}\right]_{v}\right\rangle$ is equal to

$$
c_{k_{1}, k_{2}, M_{1}, M_{2}, g ; v} \sum_{\substack{n \in \mathbb{Z}, r \in \mathbb{Z}^{g} \\ 4 n \geq M_{1}^{-1}\left[r^{t}\right]}}\left(4 n\left|M_{1}\right|-\tilde{M}_{1}\left[r^{t}\right]\right)^{v} \overline{b(n, r)}\left\langle F, P_{k, M ;(n, r)}\right\rangle,
$$

where $c_{k_{1}, k_{2}, M_{1}, M_{2}, g ; v}$ is as in (3.6). Note that if $4 n>M_{1}^{-1}\left[r^{t}\right]$, then $4 n>M^{-1}\left[r^{t}\right]$, and hence the Poincaré series $P_{k, M ;(n, r)}$ are all cusp forms. On the other hand, if $4 n=M_{1}^{-1}\left[r^{t}\right]$, then $4 n \geq M^{-1}\left[r^{t}\right]$ implies that $r=0$ and $n=0$, in which case one has the Eisenstein series $E_{k, M}$. Since $F$ is a cusp form, $\left\langle F, E_{k, M}\right\rangle$ is zero. From (2.1), it follows that $\left\langle F,\left[G, E_{k_{2}, M_{2}}\right]_{v}\right\rangle$ is equal to

$$
c_{k, k_{2}, M, M_{2}, g ; v} \sum_{\substack{n \in \mathbb{Z}, r \in \mathbb{Z}^{g} \\ 4 n \geq M_{1}^{-1}\left[r^{t}\right]}} \frac{\left(4 n\left|M_{1}\right|-\tilde{M}_{1}\left[r^{t}\right]\right)^{v} a(n, r) \overline{b(n, r)}}{\left(4 n|M|-\tilde{M}\left[r^{t}\right]\right)^{\kappa}}
$$

where $c_{k, k_{2}, M, M_{2}, g ; v}$ is defined as in (3.4).

\section{Acknowledgements}

The authors thank the referee for useful suggestions. Most of this work was done when the second author was a graduate student at the Harish-Chandra Research Institute, Allahabad, and he wishes to thank HRI for its support. 


\title{
References
}

[1] S. Böcherer and W. Kohnen, 'Estimates for Fourier coefficients of Siegel cusp forms', Math. Ann. 297 (1993), 499-517.

[2] Y. Choie, 'Jacobi forms and the heat operator', Math. Z. 225(1) (1997), 95-101.

[3] Y. Choie, 'Jacobi forms and the heat operator II', Illinois J. Math. 42 (1998), 179-186.

[4] Y. Choie and H. Kim, 'Differential operators on Jacobi forms of several variables', J. Number Theory 82 (2000), 140-163.

[5] Y. Choie and W. Kohnen, 'Rankin's method and Jacobi forms', Abh. Math. Sem. Univ. Hamburg 67 (1997), 307-314.

[6] H. Cohen, 'Sums involving the values at negative integers of $L$-functions of quadratic characters', Math. Ann. 217 (1977), 81-94.

[7] M. Eichler and D. Zagier, The Theory of Jacobi Forms, Progress in Mathematics, 55 (Birkhäuser, Boston, MA, 1985).

[8] W. Kohnen, 'On the uniform convergence of Poincaré series of exponential type on Jacobi groups', Abh. Math. Sem. Univ. Hamburg 66 (1996), 131-134.

[9] H. Maass, 'Über die gleichmässige Konvergenz der Poincaréschen Reihen $n$-ten Grades', Nachr. Akad. Wiss. Göttingen Math.-Phys. Kl. II 12 (1964), 137-144.

[10] R. A. Rankin, 'The construction of automorphic forms from the derivatives of a given form', J. Indian Math. Soc. 20 (1956), 103-116.

[11] R. A. Rankin, 'The construction of automorphic forms from the derivatives of given forms', Michigan Math. J. 4 (1957), 181-186.

[12] B. Sahu, 'Some problems in number theory', PhD Thesis, Harish-Chandra Research Institute, University of Allahabad, 2008.

[13] D. Zagier, 'Modular forms whose Fourier coefficients involve zeta-functions of quadratic fields', in: Modular Functions of One Variable, VI (Proc. 2nd Int. Conf., Univ. Bonn, Bonn, 1976), Lecture Notes in Mathematics, 627 (Springer, Berlin, 1977), pp. 105-169.

[14] D. Zagier, 'Modular forms and differential operators', Proc. Indian Acad. Sci. Math. Sci. 104 (1994), 57-75.

[15] C. Ziegler, 'Jacobi forms of higher degree', Abh. Math. Sem. Univ. Hamburg 59 (1989), 191-224.

\author{
B. RAMAKRISHNAN, Harish-Chandra Research Institute, Chhatnag Road, \\ Jhusi, Allahabad 211 019, India \\ e-mail: ramki@hri.res.in
}

BRUNDABAN SAHU, School of Mathematical Sciences, University College Dublin, Belfield, Dublin 4, Ireland

e-mail: brundaban.sahu@ucd.ie 